\title{
CORRECTION
}

\section{Correction: HSP70L1-mediated intracellular priming of dendritic cell vaccination induces more potent CTL response against cancer}

Shuxun Liu, Lin Yi, Ling Ma, Jinxia Jiang, Lijun Song, Juan Liu and Xuetao Cao

Cellular \& Molecular Immunology (2020) 17:108-109; https://doi.org/10.1038/s41423-019-0335-9

Correction to: Cellular \& Molecular Immunology https://doi.org/ 10.1038/cmi.2016.33, published online 27 June 2016.

Shuxun Liu, Lin Yi, Ling Ma, Jinxia Jiang, Lijun Song, Juan Liu, and Xuetao Cao HSP70L1-mediated intracellular priming of dendritic cell vaccination induces more potent CTL response against cancer. Cell Mol Immunol. 2018 Feb;15(2):135-145.
In the published version of Fig. 3D, the data of the $\mathrm{CH}$ and AdCtrl groups in the CFSE-Lovo/Medium panel were mistakenly presented with incorrected images. Figure 3D has now been corrected. The corrected version of Figure 3 is shown below.

Although we regret our mistake during figure assembly and would like to apologize for any inconvenience it may have caused, we did not manipulate our data in any way. This unintentional error also has no bearing on the work's scientific conclusions. 
a

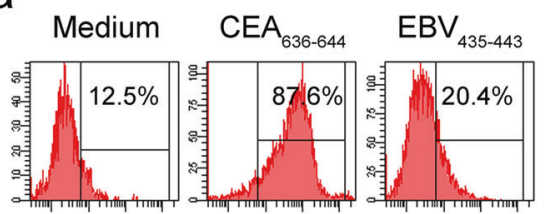

$\beta 2 m+$

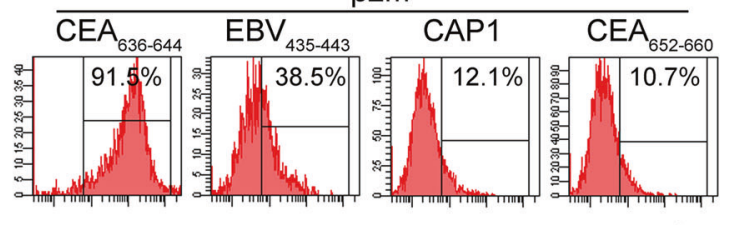

HLA-A11

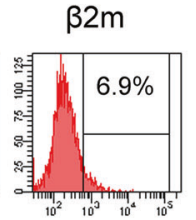

b

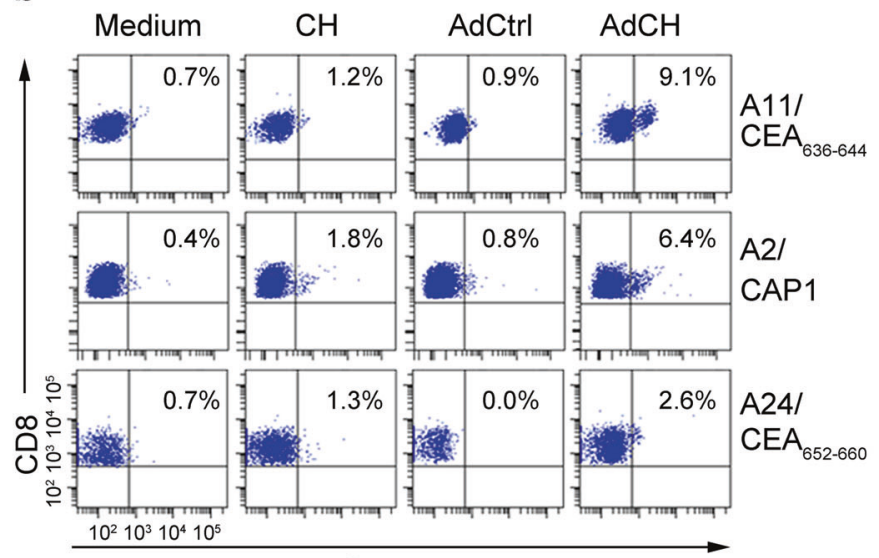

Pentamer

C
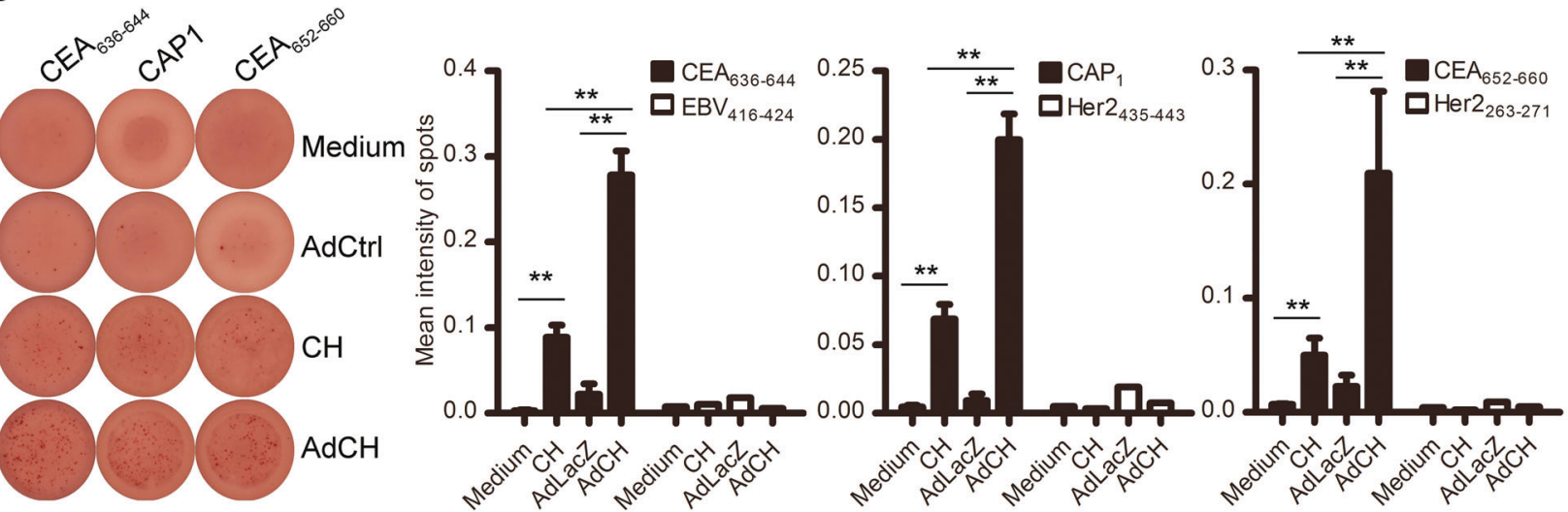

d
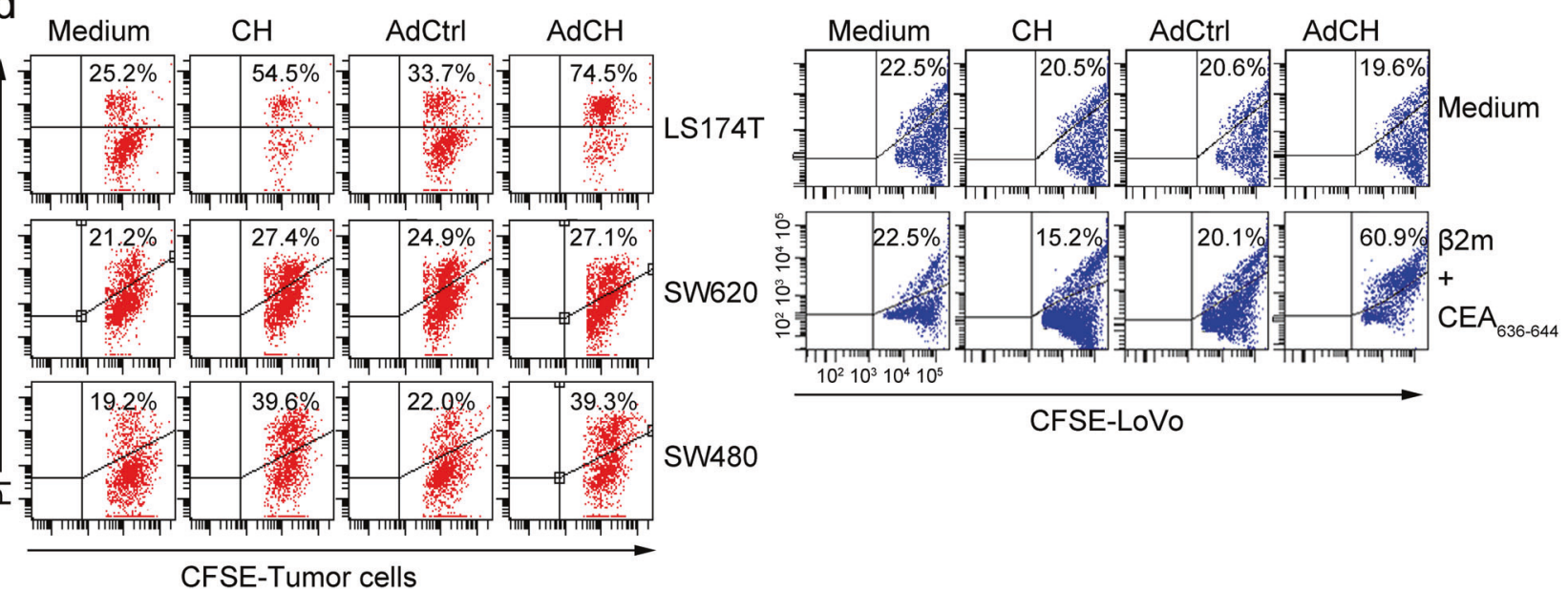

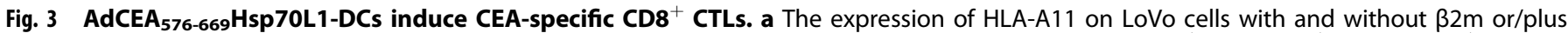
indicated nanopeptide $(1 \mathrm{\mu g} / \mathrm{ml})$ was detected using FACS. b, c Immature DCs, respectively, from HLA-A $11^{+}, \mathrm{HLA}_{-} \mathrm{A} 2.1^{+}$or HLA-A24 ${ }^{+}$healthy donors were pulsed with $\mathrm{CEA}_{576-669} \mathrm{HSP70L1}(\mathrm{CH})$ or transfected with the indicated Ad for $48 \mathrm{~h}$, and then restimulated with autogenous $\mathrm{CD}^{+} \mathrm{T}$ cells for a total of three cycles; then the frequencies of $\mathrm{CD} 8^{+} \mathrm{T}$ cells specifically recognizing epitopes of HLA-A11-resricted CEA $636-644, \mathrm{HLA}-$ A2-restricted CAP1 or HLA-A24-restricted CEA ${ }_{652-660}$ were detected, respectively, using Pentamer staining and FACS analysis (b) or IFN- $\gamma /$ ELISPOT (c), and the cytotoxicity by autogenous CD8 ${ }^{+} T$ cells to LS-174T, SW480, SW620 or LoVo tumor cells labeled by CFSE was evaluated using cytotoxic assays and FACS analysis (d). Representative blots of IFN- $\gamma /$ ELISPOT (c, left); HLA-24.2-restricted Her2 $263-271$, HLA-A2.1-restricted Her2 $435-443$, and HLA-A11.1-restricted $\mathrm{EBV}_{416-424}$ were used as negative control in (c) (right three panels). All results are representative of three independent experiments. Values are $\%$ in FACS graphs $(\mathbf{a}, \mathbf{b}, \mathbf{d})$, and mean \pm s.d. of three determinants (c, below). One-way ANOVA (c). ${ }^{* *} P<0.01$. 